\title{
A new chapter
}

\section{Arnold G Vulto}

Welcome to the new generation EJHP, now in its 19th volume, with our new partners, the BMJ Group. The EAHP board selected the BMJ Group to realise the high ambitions that the European Association of Hospital Pharmacists has with its Journal. To mention a few: to create a peer reviewed academic journal supported by educational and practical articles, to support the rich educational content with accredited e-learning modules, to improve the synergy with the other educational activities of EAHP such as the annual Congress and the European Academy of Hospital Pharmacy seminars and summits.

With this volume, the two previous journals, EJHP Practice and EJHP Science have been merged to create the new title European Journal of Hospital Pharmacy: Science and Practice. Each issue will therefore contain a mixture of peer reviewed scientific content and peer reviewed professional and practical content. It is hoped that EAHP members will continue to contribute with updates on research projects carried out by our members and other professionals in the pharmaceutical sciences. With the high quality papers that we receive from you, and supported by a highly reputable publisher, we hope to be indexed in ISI and PubMed in a shorter time period (2-3 years), to fulfil another long- standing EAHP ambition.

Each issue will continue to have interesting news for our members, a cover story, commentaries, medication safety forum and so on: so I hope you will continue to find your favourite sections. To improve the quality, more content is now externally peer reviewed. I am happy that all editorial board members have agreed to support the new journal. For the peer reviewed scientific content, my colleague, Professor Per Honoré Hartvig from Copenhagen

\section{Correspondence to Arnold G Vulto,} Editor-in-Chief EJHP Practice \& Science, Department of Hospital Pharmacy, Erasmus University Medical center, Rotterdam 3015 CE, The Netherlands; editor.ejhp@bmjgroup.com takes responsibility, supported by Dr. V'lain Fenton May who has been involved in the journal almost from its inception. The more practice oriented papers are initially scrutinised by Dr. Jean Vigneron from Nancy (France) and myself. You can find the profiles of the editorial team on page 7 . We welcome any feedback, and especially any suggestions for improvement or topical areas that you would like us to focus on in future issues.

The focus of this issue is on palliative pharmaceutical care, typically a topic neglected in most undergraduate curricula, and reflected in medical practice in general. Much of our practice is directed at acute care, however, patients with serious chronic conditions beyond cure or who have a terminal disease also need our full attention. From several surveys carried out by the European Society for Medical Oncology (ESMO) and the European Association of Palliative Care (EAPC), we have recently learned that, for instance, the availability of adequate pain treatment (sometimes due to regulatory barriers) is far from ideal. One of those surveys is being summarised by Dr Nathan Cherny ${ }^{1}$. He writes: "cancer pain remains an issue, assessment is often poor and suboptimal treatment and outcomes are frequent'. It is obvious: there is much room for improvement, and hospital pharmacists are in an ideal situation to improve that situation. Dr Cherny makes nine recommendations in his article, and some of them are an open invitation for hospital pharmacists to take action to improve this poor situation. We can educate doctors and nursing staff on the proper use of analgesics like opiods, to teach them that there are no reasons to be reluctant in using them. We can also take the initiative for simple protocols for adequate pain control with opiods (recommendation 8). We were very fortunate to have Prof Franco De Conno from Milan as our guest editor of this cover story. Prof De Conno is one of the founders of the EAPC and through his support, several authors were stimulated to write for us. I wish to express my gratitude to Prof De Conno for his help in assembling and reviewing this cover story.

The official journal of the European Association of Hospital Pharmacists is a journal from and for EAHP members. We can only succeed in our mission with your support. Therefore it is important that you share with us your ideas, and hopefully also articles describing achievements or developments in your practice. If you are engaged in research, then EJHP should be your prime platform to share the results with those who matter: your fellow hospital pharmacists. The aim of research is to improve hospital pharmacy practice, so it is imperative to publish your research results in a journal that will reach all of your colleagues in hospital pharmacy. The EJHP is unique as it will continue to be distributed to all EAHP members during 2012, meaning all members of national associations that are federated in EAHP.

There is no competitor, there is no alternative!

Only by sharing the best ideas and practices with each other can we create a patient care environment aimed at optimal and safe specialised pharmaceutical care.

The papers we present in this issue's cover story on palliative pharmaceutical care are just of a beginning, but we hope they are a helpful starting point to improve the care for your patients. Finally, whatever may change in the journal, its main purpose will always be to support you in providing the best of care for your patients.

Competing interests None.

Provenance and peer review Not commissioned; not internally peer reviewed.

Accepted 26 January 2012

European Journal of Hospital Pharmacy

2012;19:1

doi:10.1136/ejhpharm-2012-000083

\section{Reference}

1. Cherny N. How well are we doing in treating cancer pain in Europe: Key findings of The European Pain in Cancer (EPIC) Report. European Journal of Hospital Pharmacy 2012;19:30-32. 